\title{
Self-similar extrapolation from weak to strong coupling
}

\author{
S. Gluzman ${ }^{1}$ and V.I. Yukalov ${ }^{2}$ \\ ${ }^{1}$ Generation 5 Mathematical Technologies Inc., Corporate Headquarters, \\ 515 Consumer Road, Toronto, Ontario M2J 4Z2, Canada \\ and \\ ${ }^{2}$ Bogolubov Laboratory of Theoretical Physics, \\ Joint Institute for Nuclear Research, Dubna 141980, Russia
}

\begin{abstract}
The problem is addressed of defining the values of functions, whose variables tend to infinity, from the knowledge of these functions at asymptotically small variables close to zero. For this purpose, the extrapolation by means of different types of self-similar approximants is employed. Two new variants of such an extrapolation are suggested. The methods are illustrated by several examples of systems typical of chemical physics, statistical physics, and quantum physics. The developed methods make it possible to find good approximations for the strong-coupling limits from the knowledge of the weak-coupling expansions.
\end{abstract}

Keywords Weak-coupling expansions. Asymptotic series. Extrapolation of asymptotic series. Strong-coupling limit. Self-similar approximation theory. 


\section{Introduction}

In a variety of problems of chemistry and physics [1], there is the necessity of defining the properties of systems in the strong-coupling limit, when, however, this limit cannot be accessed directly, but when, because of the complexity of the problem, only the weakcoupling expansion is available. Then the principal question is whether one can infer the strong-coupling properties from a weak-coupling expansion?

In the present paper, we address this principal question and show that the problem can be resolved by employing the self-similar approximation theory [2-12] allowing for the extrapolation of asymptotic series to finite values of expansion parameters. Here we concentrate on the question of how to better organize an extrapolation not merely to finite parameter values, but to their infinite values. We show that the self-similar extrapolation is a convenient tool for this purpose. Since this approach allows for the construction of different approximation schemes, it is useful to compare the latter in order to decide which of them better suits the posed problem. It is also necessary to make some modifications for adjusting the methods to the case, when the behavior at infinity is of interest. Here we make such modifications and suggest two new ways of constructing self-similar approximants, which have not been analyzed in our previous publications.

To illustrate the applicability of the developed methods and to compare their accuracy, we consider several problems whose mathematical structure is typical of chemical physics, quantum physics, and statistical physics. We compare different variants of the self-similar approximants. Also, we consider the Padé approximants [13] and show that the accuracy of the best Padé approximant is always inferior to that of the best self-similar approximant. In some cases, the Padé approximants are not applicable at all, yielding qualitatively wrong results, while there always exists an accurate self-similar approximant.

In Sec. 2, we give the general formulation of the problem, defining the strong-coupling exponents and amplitudes. We introduce two novel methods of constructing the self-similar approximants yielding the corrected self-similar approximants and the iterated root approximants. For the beginning, we choose, in Sec. 3, the Debye-Hückel function from the theory of strong electrolytes. The quartic anharmonic oscillator of Sec. 4 provides the weak-coupling asymptotic series with the mathematical structure that is common for many problems of quantum mechanics and field theory. In Sec. 5, we consider the expansion factor of a polymer chain. The ground-state energy for a model of one-dimensional string is calculated in Sec. 6. A more realistic model of fluctuating fluid membrane is treated in Sec. 7. A dilute gas of strongly interacting fermions is studied in Sec. 8. Then, in Sec. 9, the strong-coupling limit of a one-dimensional Bose system is analyzed. The problem of calculating the BoseEinstein condensation temperature of an interacting Bose gas is investigated in Sec. 10. Finally, Sec. 11 concludes.

Some of the examples, analized in the present paper, have been considered earlier $[14,15]$, however, by other methods. The main difference of this paper from our previous publications is threefold: (i) We consider a large variety of problems to show the generality of our theory. (ii) We give a comparative analysis of different approximants. (iii) We suggest new variants of self-similar approximants, which have not been considered previously. 


\section{Formulation of general problem}

Let us be interested in a real function $f(g)$ of a real coupling parameter $g$, when $g \rightarrow$ $\infty$, which corresponds to the strong-coupling limit. But assume that the problem is so complicated that we are not able to calculate this limit directly. Instead, we can get only the weak-coupling expansion, when perturbation theory is applicable. Then, we can find the value

$$
f(g) \simeq f_{k}(g) \quad(g \rightarrow 0)
$$

for asymptotically small $g$, which is given by the weak-coupling expansion

$$
f_{k}(g)=f_{0}(g) \sum_{n=0}^{k} a_{n} g^{n} .
$$

Here the factor $f_{0}(g)$ is not expandable in powers of $g$. Without the loss of generality, we can always set $a_{0}=1$, which, however, is not principal.

\subsection{Strong-coupling exponents and amplitudes}

¿From some additional information, we may know that the behavior of the sought function at large coupling parameter is as follows:

$$
f(g) \simeq B g^{\beta} \quad(g \rightarrow \infty) .
$$

The power index $\beta$ is called the strong-coupling exponent and can be defined as

$$
\beta \equiv \lim _{g \rightarrow \infty} \frac{\ln f(g)}{\ln g} .
$$

This can also be written as

$$
\beta=\lim _{g \rightarrow \infty} \frac{d \ln f(g)}{d \ln g}=\lim _{g \rightarrow \infty} \frac{g}{f(g)} \frac{d f(g)}{d g} .
$$

We assume that this exponent is known, and what we need to find is the strong-coupling amplitude

$$
B \equiv \lim _{g \rightarrow \infty} \frac{f(g)}{g^{\beta}} .
$$

The problem we confront is how to find the strong-coupling amplitude (5) from the knowledge of the weak-coupling expansion (2)?

\subsection{Corrected self-similar approximants}

An effective extrapolation can be done by means of the self-similar approximation theory [212]. Then, from the weak-coupling expansion $f_{k}(g)$, we construct a self-similar approximant $f_{k}^{*}(g)$. When the behavior of the sought function is supposed to be exponential, we have to use the self-similar exponential approximants $[16,17]$. But in the considered case (3) of the power law behavior, it is better to employ the self-similar factor approximants [18-21]. 
Suppose, we have found an approximant $f_{k}^{*}(g)$. This can be an exponential approximant $[16,17]$ or a factor approximant [18-21]. Now we suggest a novel way of correcting this approximant and, respectively, improving the accuracy of calculating the strong-coupling amplitude.

Let an approximant $f_{k}^{*}(g)$ be given, whose parameters are defined by the accuracythrough-order procedure, which implies that the approximant $f_{k}^{*}(g)$ is re-expanded in powers of $g$ and compared, term by term, with the initial expansion, so that

$$
f_{k}^{*}(g) \simeq f_{k}(g) \quad(g \rightarrow 0) .
$$

This is also called the re-expansion procedure. More details, describing this procedure, can be found in Ref. [15].

In constructing the approximant $f_{k}^{*}(g)$, we keep in mind the limiting condition

$$
\lim _{g \rightarrow \infty} \frac{\ln f_{k}^{*}(g)}{\ln g}=\beta .
$$

Our aim is to find the strong-coupling amplitude

$$
B_{k} \equiv \lim _{g \rightarrow \infty} \frac{f_{k}^{*}(g)}{g^{\beta}} .
$$

Now, let us introduce a correcting function

$$
C_{k+p}(g) \simeq \frac{f_{k+p}(g)}{f_{k}^{*}(g)} \quad(g \rightarrow 0),
$$

which is defined as an expansion in powers of $g$, such that

$$
C_{k+p}(g)=\sum_{n=0}^{k+p} b_{n} g^{n},
$$

whose coefficients $b_{n}$ are, evidently, functions of the initial coefficients $a_{n}$ from series (2). Since the parameters of $f_{k}^{*}(g)$, according to Eq. (6), are defined through the re-expansion procedure, the correcting function (10) contains not all powers of $g$ but only those starting from the order $k+1$, that is,

$$
C_{k+p}(g)=1+\sum_{n=k+1}^{k+p} b_{n} g^{n} .
$$

This fact essentially simplifies the construction of a self-similar approximant $C_{k+p}^{*}(g)$ from the correcting function (11). Constructing the approximant $C_{k+p}^{*}(g)$, we impose the limiting condition

$$
\lim _{g \rightarrow \infty} C_{k+p}^{*}(g)=\text { const } .
$$

The corrected self-similar approximant is defined as

$$
\widetilde{f}_{k+p}(g) \equiv f_{k}^{*}(g) C_{k+p}^{*}(g) .
$$


Because of condition (12),

$$
\lim _{g \rightarrow \infty} \frac{\ln C_{k+p}^{*}(g)}{\ln g}=0 .
$$

Therefore the strong-coupling exponent does not change,

$$
\lim _{g \rightarrow \infty} \frac{\ln \widetilde{f}_{k+p}(g)}{\ln g}=\lim _{g \rightarrow \infty} \frac{\ln f_{k}^{*}(g)}{\ln g}=\beta .
$$

But the corrected strong-coupling amplitude

$$
\widetilde{B}_{k+p} \equiv \lim _{g \rightarrow \infty} \frac{\widetilde{f}_{k+p}(g)}{g^{\beta}}
$$

changes to

$$
\widetilde{B}_{k+p}=B_{k} \lim _{g \rightarrow \infty} C_{k+p}^{*}(g)
$$

where $B_{k}$ is given by Eq. (8).

\subsection{Iterated root approximants}

In addition to self-similar exponential approximants [16,17] and factor approximants [1821], there are self-similar root approximants [10-12]. The latter serve as a convenient tool for interpolating functions whose strong-coupling behavior is described by means of known strong-coupling expansions, when the root approximants can be uniquely determined [22]. However, the parameters of the root approximants cannot be uniquely defined through the re-expansion procedure in the region of weak coupling. Here we suggest a method allowing us to derive the parameters of the root approximants by means of the re-expansion procedure (6).

The idea of the method, allowing for a unique derivation of the parameters of the root approximants, is as follows. In order to remove the multiplicity of solutions, arising in the reexpansion procedure applied to the general form of the root approximants, it is necessary to impose some restrictions on the definition of the related parameters. Thus, we can keep the same parameters of the lower-order approximants in the higher-order approximants, leaving there unknown only the highest-order parameter that is to be found from the re-expansion procedure. And the highest-order power has to be such that to satisfy the limiting condition (7). Since in this method, the lower-order root approximants are inserted into the higherorder approximants, the resulting expressions can be called, iterated root approximants.

To be concrete, let us assume that the factor $f_{0}(g)$ possesses the strong-coupling behavior as

$$
f_{0}(g) \simeq A g^{\alpha} \quad(g \rightarrow \infty)
$$

The first-order root approximant

$$
R_{1}(g)=f_{0}(g)\left(1+A_{1} g\right)^{\gamma}
$$

where we set

$$
\gamma=\beta-\alpha,
$$


is the same as the first-order factor approximant and it is uniquely defined with $A_{1}=a_{1} / \gamma$. In the second-order root approximant

$$
R_{2}(g)=f_{0}(g)\left(\left(1+A_{1} g\right)^{2}+A_{2} g^{2}\right)^{\gamma / 2},
$$

we keep the same $A_{1}$ as before, and $\gamma$ is given by Eq. (20). Hence, we need to find only $A_{2}$ from the re-expansion procedure. In the third-order root approximant

$$
R_{3}(g)=f_{0}(g)\left(\left(\left(1+A_{1} g\right)^{2}+A_{2} g^{2}\right)^{3 / 2}+A_{3} g^{3}\right)^{\gamma / 3},
$$

the parameters $A_{1}$ and $A_{2}$ are kept the same as in the previous expression (21), while $A_{3}$ is defined through the re-expansion procedure. Such an iteration construction continues, with the general $k$-approximant being

$$
R_{k}(g)=f_{0}(g)\left(\left(\ldots\left(1+A_{1} g\right)^{2}+A_{2} g^{2}\right)^{3 / 2}+\ldots+A_{k} g^{k}\right)^{\gamma / k},
$$

where all parameters $A_{n}$, with $n=1,2, \ldots, k-1$ are the same as in the $k$-1-order approximant and the parameter $A_{k}$ is defined by the re-expansion procedure.

Constructing in this way the iterated root approximants yields the strong-coupling amplitudes

$$
B_{k}=\lim _{g \rightarrow \infty} \frac{R_{k}(g)}{g^{\beta}} .
$$

In particular,

$$
B_{1}=A A_{1}^{\gamma}, \quad B_{2}=A\left(A_{1}^{2}+A_{2}\right)^{\gamma / 2}, \quad B_{3}=A\left(\left(A_{1}^{2}+A_{2}\right)^{3 / 2}+A_{3}\right)^{\gamma / 3},
$$

and so on, with the $k$-order amplitude

$$
B_{k}=A\left(\left(\ldots\left(A_{1}^{2}+A_{2}\right)^{3 / 2}+A_{3}\right)^{4 / 3}+\ldots+A_{k}\right)^{\gamma / k} .
$$

An accurate evaluation of the strong-coupling amplitude is the main aim of the suggested scheme. In the following sections, we shall consider several examples of explicit calculations of strong-coupling amplitudes, comparing the accuracy of different approaches.

\section{Debye-Hückel function}

Let us start with the case, when the sought function is actually known. This will allow us to easily determine the accuracy of each scheme. Let us consider the Debye-Hückel function that is met in the theory of strong electrolites [23,24]. In dimensionless units, the function reads as

$$
f(g)=\frac{2}{g}-\frac{2}{g^{2}}\left(1-e^{-g}\right) .
$$

In view of Eqs. (2)-(5), we have $f_{0}(g)=1, \beta=-1$, and

$$
B=2 \text {. }
$$


The coefficients, corresponding to the weak-coupling expansion (2), are

$$
\begin{gathered}
a_{1}=-\frac{1}{3}, \quad a_{2}=\frac{1}{12}, \quad a_{3}=-\frac{1}{60}, \\
a_{4}=\frac{1}{360}, \quad a_{5}=-\frac{1}{2520}, \quad a_{6}=\frac{1}{20160}, \quad a_{7}=-\frac{1}{181440} .
\end{gathered}
$$

The Padé approximants do not provide good accuracy. The best of them,

$$
P_{3 / 4}(g)=\frac{1+c_{1} g+c_{2} g^{2}+c_{3} g^{3}}{1+c_{4} g+c_{5} g^{2}+C_{6} g^{3}+c_{7} g^{4}},
$$

possessing the correct strong-coupling exponent $\beta=-1$, and invoking all seven expansion coefficients $a_{n}$, gives the strong-coupling amplitude

$$
B_{3 / 4}=\lim _{g \rightarrow \infty} g P_{3 / 4}(g)=\frac{c_{3}}{c_{7}}=1.6
$$

with an error $\varepsilon\left(B_{3 / 4}\right)=-20 \%$.

\subsection{Self-similar factor approximants}

The factor approximant, using the expansion terms up to the third order, that is, involving the coefficients $a_{1}, a_{2}, a_{3}$, reads as

$$
f_{3}^{*}(g)=\left(1+A_{1} g\right)^{n_{1}}\left(1+A_{2} g\right)^{n_{2}},
$$

with the condition

$$
n_{1}+n_{2}=-1 .
$$

This gives the strong-coupling amplitude

$$
B_{3}=A_{1}^{n_{1}} A_{2}^{n_{2}}=1.64,
$$

whose error is $\varepsilon\left(B_{3}\right)=-18 \%$.

The fifth-order factor approximant

$$
f_{5}^{*}(g)=\left(1+A_{1} g\right)^{n_{1}}\left(1+A_{2} g\right)^{n_{2}}\left(1+A_{3} g\right)^{n_{3}},
$$

invoking the weak-coupling expansion terms up to the fifth order in $g$ and satisfying the condition

$$
n_{1}+n_{2}+n_{3}=-1,
$$

yields the strong-coupling amplitude

$$
B_{5}=A_{1}^{n_{1}} A_{2}^{n_{2}} A_{3}^{n_{3}}=2.295,
$$

with an error $\varepsilon\left(B_{5}\right)=15 \%$.

Note that the parameters of each of the factor approximants are defined through the re-expansion procedure. So, the values of $A_{i}$ and $n_{i}$ in each order are different. We use the same letters in different approximants just for the simplicity of notation. 
The factor approximant, using all seven expansion terms, is

$$
f_{7}^{*}(g)=\left(1+A_{1} g\right)^{n_{1}}\left(1+A_{2} g\right)^{n_{2}}\left(1+A_{3} g\right)^{n_{3}}\left(1+A_{4} g\right)^{n_{4}},
$$

under the condition

$$
n_{1}+n_{2}+n_{3}+n_{4}=-1 .
$$

This gives the strong-coupling amplitude

$$
B_{7}=A_{1}^{n_{1}} A_{2}^{n_{2}} A_{3}^{n_{3}} A_{4}^{n_{4}}=1.799,
$$

with an error $\varepsilon\left(B_{7}\right)=-10 \%$.

\subsection{Corrected factor approximants}

Following the scheme of Sec. 2, we can define the corrected factor approximants. To this end, let us introduce the correction function

$$
C_{7}(g) \simeq \frac{f_{7}(g)}{f_{3}^{*}(g)} \quad(g \rightarrow 0)
$$

which yields

$$
C_{7}(g)=1+b_{4} g^{4}+b_{5} g^{5}+b_{6} g^{6}+b_{7} g^{7} .
$$

¿From this expansion, we construct the factor approximant

$$
C_{7}^{*}(g)=1+b_{4} g^{4}\left(1+D_{3} g\right)^{n_{3}}\left(1+D_{4} g\right)^{n_{4}},
$$

under the condition

$$
4+n_{3}+n_{4}=0 .
$$

The corrected factor approximant is defined as

$$
\tilde{f}_{7}(g)=f_{3}^{*}(g) C_{7}^{*}(g) .
$$

This yields the strong-coupling amplitude

$$
\widetilde{B}_{7}=B_{3}\left(1+b_{4} D_{3}^{n_{3}} D_{4}^{n_{4}}\right)=1.944,
$$

with an error $\varepsilon\left(\widetilde{B}_{7}\right)=-2.8 \%$.

\subsection{Simple root approximants}

A simple root approximant

$$
r_{3}^{*}(g)=\left(\left(1+A_{1} g\right)^{n_{1}}+A_{2} g^{2}\right)^{-1 / 2}
$$

in which the parameters are defined trough the re-expansion procedure, gives the strongcoupling amplitude

$$
B_{3}=\frac{1}{\sqrt{A_{2}}}=2.164
$$

whose error is $\varepsilon\left(B_{3}\right)=8.2 \%$. 


\subsection{Corrected root approximants}

The above simple root approximant can be corrected according to Sec. 2. For this purpose, we define the correction function

$$
C_{7}(g) \simeq \frac{f_{7}(g)}{r_{3}^{*}(g)} \quad(g \rightarrow 0),
$$

corresponding to the expansion in powers of $g$ up to the seventh order, and construct the root approximant

$$
C_{7}^{*}(g)=1+b_{4} g^{4}\left(\left(1+D_{3} g\right)^{n_{3}}+D_{4} g^{2}\right)^{-2} .
$$

Then the corrected root approximant is

$$
r_{7}^{*}(g)=r_{3}^{*}(g) C_{7}^{*}(g) .
$$

The latter gives the strong-coupling amplitude

$$
\widetilde{B}_{7}=\frac{1}{\sqrt{A_{2}}}\left(1+\frac{b_{4}}{D_{4}^{2}}\right)=1.875,
$$

with an error $\varepsilon\left(\widetilde{B}_{7}\right)=-6.3 \%$.

\subsection{Iterated root approximants}

The iterated root approximants, according to Sec. 2, have the form

$$
R_{k}(g)=\left(\left(\ldots\left(1+A_{1} g\right)^{2}+A_{2} g^{2}\right)^{3 / 2}+\ldots+A_{k} g^{k}\right)^{\beta / k},
$$

where $\beta=-1$. Therefore, the strong-coupling amplitude of $k$-order is

$$
B_{k}=\left(\left(\ldots\left(A_{1}^{2}+A_{2}\right)^{3 / 2}+A_{3}\right)^{4 / 3}+\ldots+A_{k}\right)^{\beta / k} .
$$

¿From here, we find

$$
\begin{gathered}
B_{1}=3, \quad B_{2}=2.449, \quad B_{3}=2.229, \quad B_{4}=2.127, \\
B_{5}=2.067, \quad B_{6}=2.032, \quad B_{7}=2.009 .
\end{gathered}
$$

The seventh order amplitude has an error $\varepsilon\left(B_{7}\right)=0.45 \%$. Thus, the iterated root approximant (49) of the seven-th order is the most accurate among all above considered approximants.

\section{Quartic anharmonic oscillator}

The anharmonic oscillator, with the Hamiltonian

$$
H=-\frac{1}{2} \frac{d^{2}}{d x^{2}}+\frac{1}{2} x^{2}+g x^{4},
$$


is the model imitating many systems in quantum chemistry, atomic physics, condensedmatter physics, and field theory. Here, $g$ is the coupling parameter and we set $x \in(-\infty, \infty)$ and $g \in[0, \infty)$. The ground-state energy for this Hamiltonian, found by means of the weak-coupling perturbation theory [25,26], when $g \rightarrow 0$, reads as

$$
e(g) \simeq a_{0}+a_{1} g+a_{2} g^{2}+a_{3} g^{3}+a_{4} g^{4}+a_{5} g^{5}+a_{6} g^{6}+a_{7} g^{7},
$$

with the coefficients

$$
\begin{gathered}
a_{0}=\frac{1}{2}, \quad a_{1}=\frac{3}{4}, \quad a_{2}=-\frac{21}{8}, \quad a_{3}=\frac{333}{16}, \\
a_{4}=-\frac{30885}{128}, \quad a_{5}=\frac{916731}{256}, \quad a_{6}=-\frac{65518401}{1024}, \quad a_{7}=\frac{2723294673}{2048} .
\end{gathered}
$$

The strong-coupling limit for the ground-state energy yields

$$
e(g) \simeq 0.667986 g^{1 / 3} \quad(g \rightarrow \infty)
$$

Hence, the strong-coupling exponent $\beta=1 / 3$ and the strong-coupling amplitude is

$$
B=0.667986
$$

As is seen, the coefficients of the weak-coupling expansion quickly grow, and perturbation theory is divergent for any nonzero $g$. The standard Padé approximants for this problem are not applicable at all, being unable to satisfy the fractional strong-coupling exponent $\beta=1 / 3$. It is possible to introduce the power-transformed approximants, similarly to Ref. [14], fixing the exponent $\beta=1 / 3$. But this way does not provide a great accuracy improvement. The best power-transformed Padé approximant gives an error of $8 \%$.

\subsection{Self-similar factor approximants}

Using the third order of the weak-coupling expansion (52), we get the self-similar factor approximant

$$
e_{3}^{*}(g)=\frac{1}{2}\left(1+A_{1} g\right)^{n_{1}}\left(1+A_{2} g\right)^{n_{2}}
$$

under the condition

$$
n_{1}+n_{2}=\frac{1}{3}
$$

This gives the strong-coupling amplitude

$$
B_{3}=\frac{1}{2} A_{1}^{n_{1}} A_{2}^{n_{2}}=0.75
$$

with an error $\varepsilon\left(B_{3}\right)=12 \%$.

The fifth-order factor approximant has the form

$$
e_{5}^{*}(g)=\frac{1}{2}\left(1+A_{1} g\right)^{n_{1}}\left(1+A_{2} g\right)^{n_{2}}\left(1+A_{3} g\right)^{n_{3}},
$$

with the condition

$$
n_{1}+n_{2}+n_{3}=\frac{1}{3}
$$


Then the strong-coupling amplitude is

$$
B_{5}=\frac{1}{2} A_{1}^{n_{1}} A_{2}^{n_{2}} A_{3}^{n_{3}}=0.725
$$

whose error is $\varepsilon\left(B_{5}\right)=8.5 \%$.

The seventh-order factor approximant is

$$
e_{7}^{*}(g)=\frac{1}{2}\left(1+A_{1} g\right)^{n_{1}}\left(1+A_{2} g\right)^{n_{2}}\left(1+A_{3} g\right)^{n_{3}}\left(1+A_{4} g\right)^{n_{4}},
$$

with the condition

$$
n_{1}+n_{2}+n_{3}+n_{4}=\frac{1}{3} .
$$

¿From here, we have the strong-coupling amplitude

$$
B_{7}=\frac{1}{2} A_{1}^{n_{1}} A_{2}^{n_{2}} A_{3}^{n_{3}} A_{4}^{n_{4}}=0.712,
$$

with an error $\varepsilon\left(B_{7}\right)=6.6 \%$.

\subsection{Corrected factor approximants}

Defining the correction function

$$
C_{7}(g) \simeq \frac{e_{7}(g)}{e_{3}^{*}(g)} \quad(g \rightarrow 0),
$$

we get the series

$$
C_{7}(g)=1+\sum_{n=4}^{7} b_{n} g^{n} .
$$

The latter generates the factor approximant

$$
C_{7}^{*}(g)=1+b_{4} g^{4}\left(1+D_{3} g\right)^{n_{3}}\left(1+D_{4} g\right)^{n_{4}},
$$

where

$$
4+n_{3}+n_{4}=0
$$

The corrected factor approximant

$$
\widetilde{e}_{7}(g)=e_{3}^{*}(g) C_{7}^{*}(g)
$$

yields the strong-coupling amplitude

$$
\widetilde{B}_{7}=\frac{1}{2} A_{1}^{n_{1}} A_{2}^{n_{2}}\left(1+b_{4} D_{3}^{n_{3}} D_{4}^{n_{4}}\right)=0.728,
$$

with an error $\left.\varepsilon\left(\widetilde{B}_{7}\right)=9 \%\right)$. 


\subsection{Simple root approximants}

Simple root approximants do not give good accuracy. For instance, the root approximant

$$
r_{5}^{*}(g)=\frac{1}{2}\left(\left(\left(1+A_{1} g\right)^{n_{1}}+A_{2} g^{2}\right)^{2}+A_{3} g^{3}\right)^{1 / 9}
$$

results if the strong-coupling amplitude

$$
B_{5}=\frac{1}{2} A_{3}^{1 / 9}=0.824
$$

whose accuracy is characterized by the error $\varepsilon\left(B_{5}\right)=23 \%$.

\subsection{Iterated root approximants}

According to Sec. 2, the iterated root approximants are given by

$$
\begin{gathered}
R_{1}(g)=\frac{1}{2}\left(1+A_{1} g\right)^{1 / 3}, \quad R_{2}(g)=\frac{1}{2}\left(\left(1+A_{1} g\right)^{2}+A_{2} g^{2}\right)^{1 / 6}, \\
R_{3}(g)=\frac{1}{2}\left(\left(\left(1+A_{1} g\right)^{2}+A_{2} g^{2}\right)^{3 / 2}+A_{3}^{3}\right)^{1 / 9},
\end{gathered}
$$

and so on. However, the approximant $R_{4}(g)$ is complex, and we limit ourselves by Eqs. (72). The related strong-coupling amplitudes are

$$
\begin{gathered}
B_{1}=\frac{1}{2} A_{1}^{1 / 3}=0.825, \quad B_{2}=\frac{1}{2}\left(A_{1}^{2}+A_{2}\right)^{1 / 6}=0.572, \\
B_{3}=\frac{1}{2}\left(\left(A_{1}^{2}+A_{2}\right)^{3 / 2}+A_{3}\right)^{1 / 9}=0.855 .
\end{gathered}
$$

Their errors are $\varepsilon\left(B_{1}\right)=24 \%, \varepsilon\left(B_{2}\right)=-14 \%, \varepsilon\left(B_{3}\right)=28 \%$.

\subsection{Power-transformed approximants}

It is possible to define the power transformation [14] as

$$
P_{k}(g, m) \simeq f_{k}^{m}(g) \quad(g \rightarrow 0)
$$

producing the series

$$
P_{k}(g, m)=\sum_{n=0}^{k} b_{n}(m) g^{n} .
$$

Then, constructing a self-similar approximation on the basis of this series, we get $P_{k}^{*}(g, m)$. The final answer is given by the inverse power transformation

$$
f_{k}^{*}(g)=\left[P_{k}^{*}(g, m)\right]^{1 / m} .
$$

This way was analized in Ref. [14], where the transformation power $m$ was defined by variational optimization. Such a way, however, is rather cumbersome and yields the accuracy improvement not better than the simpler methods considered here.

Among the methods, studied in this Section, the self-similar factor approximants provide the best accuracy for the strong-coupling amplitude. 


\section{Polymer expansion factor}

The expansion factor $\alpha(g)$, as a function of the coupling parameter $g$, for a polymer chain with excluded interactions, can be calculated by means of perturbation theory [27] resulting in the series

$$
\alpha(g)=1+a_{1} g+a_{2} g^{2}+a_{3} g^{3}+a_{4} g^{4}++a_{5} g^{5}+a_{6} g^{6},
$$

with the coefficients

$$
\begin{gathered}
a_{1}=\frac{4}{3}, \quad a_{2}=-2.075385396, \quad a_{3}=6.296879676, \\
a_{4}=-25.05725072, \quad a_{5}=116.134785, \quad a_{6}=-594.71663 .
\end{gathered}
$$

Numerical fitting $[27,28]$ gives the phenomenological formula

$$
\alpha(g)=\left(1+7.524 g+11.06 g^{2}\right)^{0.1772} .
$$

This implies that the strong-coupling exponent $\beta=0.3544$, which is in agreement with other numerical simulations [29], where the exponent

$$
\nu \equiv \frac{1}{2}\left(1+\frac{\beta}{2}\right)
$$

was calculated, giving $\nu=0.5877$. Therefore the strong-coupling amplitude is

$$
B=1.531
$$

\subsection{Self-similar factor approximants}

The self-similar factor approximants start with the lowest order

$$
\alpha_{1}^{*}(g)=\left(1+A_{1} g\right)^{\beta},
$$

in which $A_{1}=a_{1} / \beta$ and $\beta=0.3544$. Then the strong-coupling amplitude is

$$
B_{1}=A_{1}^{\beta}=1.599,
$$

with an error $\varepsilon\left(B_{1}\right)=4.4 \%$. Increasing the approximation order improves the accuracy. Thus, the fifth-order approximant

$$
\alpha_{5}^{*}(g)=\left(1+A_{1} g\right)^{n_{1}}\left(1+A_{2} g\right)^{n_{2}}\left(1+A_{3} g\right)^{n_{3}},
$$

where

$$
n_{1}+n_{2}+n_{3}=\beta=0.3544,
$$

gives the strong-coupling amplitude

$$
B_{5}=A_{1}^{n_{1}} A_{2}^{n_{2}} A_{3}^{n_{3}}=1.541
$$

with an error $\varepsilon\left(B_{5}\right)=0.65 \%$. 


\subsection{Corrected factor approximants}

Introducing the correction function

$$
C_{3}(g) \simeq \frac{\alpha_{3}(g)}{\alpha_{1}^{*}(g)} \quad(g \rightarrow 0),
$$

and constructing the related factor approximant

$$
C_{3}^{*}(g)=1+b_{2} g^{2}\left(1+D_{1} g\right)^{-2},
$$

we come to the corrected factor approximant

$$
\widetilde{\alpha}_{3}(g)=\alpha_{1}^{*}(g) C_{3}^{*}(g) .
$$

This gives the strong-coupling amplitude

$$
\widetilde{B}_{3}=A_{1}^{\beta}\left(1+\frac{b_{2}}{D_{1}^{2}}\right)=1.552,
$$

with an error $\varepsilon\left(\widetilde{B}_{3}\right)=1.4 \%$.

The accuracy of the corrected factor approximants is close to that of the directly constructed self-similar factor approximants.

\subsection{Simple root approximants}

Simple root approximants provide sufficiently good accuracy. Thus the approximant

$$
r_{5}^{*}(g)=\left(\left(\left(1+A_{1} g\right)^{n_{1}}+A_{2} g^{2}\right)^{n_{2}}+A_{3} g^{3}\right)^{\beta / 3}
$$

yields the strong-coupling amplitude

$$
B_{5}=A_{3}^{\beta / 3}=1.537,
$$

with an error $\varepsilon\left(B_{5}\right)=0.4 \%$.

\subsection{Corrected root approximants}

For the correction function

$$
C_{5}(g) \simeq \frac{\alpha_{5}(g)}{\alpha_{1}^{*}(g)} \quad(g \rightarrow 0),
$$

the corresponding self-similar root approximant reads as

$$
C_{5}^{*}(g)=1+b_{2} g^{2}\left(\left(1+D_{1} g\right)^{m_{1}}+D_{2} g^{2}\right)^{-1} .
$$

The corrected root approximant

$$
\widetilde{r}_{5}(g)=\alpha_{1}^{*}(g) C_{5}^{*}(g)
$$

results in the strong-coupling amplitude

$$
\widetilde{B}_{5}=A_{1}^{\beta}\left(1+\frac{b_{2}}{D_{2}}\right)=1.542,
$$

whose error is $\varepsilon\left(\widetilde{B}_{5}\right)=0.72 \%$.

The simple root approximants, being already sufficiently accurate, are not improved by the corrected variants. 


\subsection{Iterated root approximants}

For the iterated root approximants, following the scheme of Sec. 2, we find the strongcoupling amplitudes

$$
\begin{array}{lll}
B_{1}=1.599, & B_{2}=1.544, & B_{3}=1.549, \\
B_{4}=1.539, & B_{5}=1.541, & B_{6}=1.537 .
\end{array}
$$

The error of the highest-order approximant is $\varepsilon\left(B_{6}\right)=0.4 \%$.

All variants of the approximants, studied in the present Section, are of about the same accuracy. We have also considered the power-transformed approximants [14], whose accuracy is found to be close to that of the iterated root approximants.

\section{$6 \quad$ Oscillating fluid string}

In biological and chemical applications, there exists an important class of membranes called fluid membranes [30]. The model of a one-dimensional fluid string, oscillating between two walls, was advanced by Edwards [31]. It has been shown [31-33] that the free energy of the string coincides with the ground-state energy of a quantum particle in a one-dimensional box. This energy, as a function of a finite wall stiffness $g$, reads as

$$
E(g)=\frac{\pi^{2}}{8 g^{2}}\left(1+\frac{g^{2}}{32}+\frac{g}{4} \sqrt{1+\frac{g^{2}}{64}}\right) .
$$

In the small-stiffness expansion

$$
E_{k}(g)=\frac{\pi^{2}}{8 g^{2}} \sum_{n=0}^{k} a_{n} g^{n}
$$

the coefficients are

$$
\begin{gathered}
a_{0}=1, \quad a_{1}=\frac{1}{4}, \quad a_{2}=\frac{1}{32}, \quad a_{3}=\frac{1}{512}, \\
a_{4}=0, \quad a_{5}=-\frac{1}{131072}, \quad a_{6}=0, \quad a_{7}=\frac{1}{16777216} .
\end{gathered}
$$

The rigid walls correspond to the stiffness $g \rightarrow \infty$, when

$$
E(\infty)=\frac{\pi^{2}}{128}=B=0.077106
$$

Hence, the strong-stiffness exponent is $\beta=0$.

Our aim is to approximate the rigid-wall limit (98) employing the small-stiffness expansion (97).

Padé approximants are not applicable for this problem, giving negative values of the strong-stiffness amplitude. So, we shall apply the self-similar approximants. 


\subsection{Simple root approximants}

The root approximants of low orders are not sufficiently accurate. Thus, the root approximant

$$
r_{3}^{*}(g)=E_{0}(g)\left(\left(1+A_{1} g\right)^{n_{1}}+A_{2} g^{2}\right),
$$

where $E_{0}(g)=\pi^{2} / 8 g^{2}$, gives the strong-stiffness amplitude

$$
B_{3}=\frac{\pi^{2}}{8} A_{2}=0.0544,
$$

with an error $\varepsilon\left(B_{3}\right)=-29 \%$. And the higher-order root approximants are not convenient, being not uniquely defined through the re-expansion procedure.

\subsection{Corrected root approximants}

As is shown in Sec. 2, we can introduce the corrected root approximants. For instance, the correction function

$$
C_{7}(g) \simeq \frac{E_{7}(g)}{r_{3}^{*}(g)} \quad(g \rightarrow 0)
$$

generates the self-similar root approximant

$$
C_{7}^{*}(g)=1+b_{4} g^{4}\left(\left(1+D_{1} g\right)^{m_{1}}+D_{2} g^{2}\right)^{-2} .
$$

Then the corrected root approximant

$$
r_{7}^{*}(g)=r_{3}^{*}(g) C_{7}^{*}(g)
$$

yields the strong-stiffness amplitude

$$
\widetilde{B}_{7}=\frac{\pi^{2}}{8} A_{2}\left(1+\frac{b_{4}}{D_{2}^{2}}\right)=0.0771,
$$

with an error $\varepsilon\left(\widetilde{B}_{7}\right)=-0.02 \%$.

We may note that the sixth-order corrected root approximant is also of good accuracy, with an error $\widetilde{B}_{6}=-0.66 \%$.

\subsection{Iterated root approximants}

The uniquely defined iterated root approximants, introduced in Sec. 2, result in the strongstiffness amplitudes

$$
\begin{gathered}
B_{1}=0.019, \quad B_{2}=0.039, \quad B_{3}=0.051, \\
B_{4}=0.058, \quad B_{5}=0.062, \quad B_{6}=0.065, \quad B_{7}=0.067 .
\end{gathered}
$$

The errors of the highest two approximations are $\varepsilon\left(B_{6}\right)=-16 \%$ and $\varepsilon\left(B_{7}\right)=-13 \%$, respectively.

Another variant was considered in Ref. [15], invoking the change of the variable $g$, and giving the strong-stiffness amplitude of seventh order with an error of $5 \%$. The best accuracy is provided by the corrected root approximant (103) yielding amplitude (104). 


\section{$7 \quad$ Fluctuating fluid membrane}

For a three-dimensional fluctuating fluid membrane, there are no exact results. Thus, the pressure of this membrane is calculated by means of perturbation theory [34] yielding expansions

$$
p_{k}(g)=\frac{\pi^{2}}{8 g^{2}} \sum_{n=0}^{k} a_{n} g^{n} .
$$

The coefficients are known [34] up to the sixth order:

$$
\begin{gathered}
a_{0}=1, \quad a_{1}=\frac{1}{4}, \quad a_{2}=\frac{1}{32}, \quad a_{3}=2.176347 \times 10^{-3}, \\
a_{4}=0.552721 \times 10^{-4}, \quad a_{5}=-0.721482 \times 10^{-5}, \quad a_{6}=-1.777848 \times 10^{-6} .
\end{gathered}
$$

The strong-stiffness limit has been found by Monte Carlo simulations [35], giving

$$
p(\infty)=0.0798 \pm 0.0003
$$

This implies that the strong-stiffness exponent is $\beta=0$ and the strong-stiffness amplitude is

$$
B=0.0798
$$

Padé approximants are inapplicable for this problem, resulting in negative values for $B$.

\subsection{Simple root approximants}

The simple root approximant

$$
r_{3}^{*}(g)=\frac{\pi^{2}}{8 g^{2}}\left(\left(1+A_{1} g\right)^{n_{1}}+A_{2} g^{2}\right)
$$

gives the strong-stiffness amplitude

$$
B_{3}=\frac{\pi^{2}}{8} A_{2}=0.056
$$

with an error $\varepsilon\left(B_{3}\right)=-30 \%$. The higher-order root approximants are not convenient to employ, since they are not uniquely defined.

\subsection{Corrected root approximants}

If we set $a_{7}=0$, we can introduce the correction function

$$
C_{7}(g) \simeq \frac{p_{7}(g)}{r_{3}^{*}(g)} \quad(g \rightarrow 0) .
$$

The corresponding self-similar root approximant is

$$
C_{7}^{*}(g)=1+b_{4} g^{4}\left(\left(1+D_{1} g\right)^{m_{1}}+D_{2} g^{2}\right)^{-2} .
$$


The corrected root approximant is defined as

$$
\widetilde{p}_{7}(g)=r_{3}^{*}(g) C_{7}^{*}(g)
$$

which gives the strong-stiffness amplitude

$$
\widetilde{B}_{7}=\frac{\pi^{2}}{8} A_{2}\left(1+\frac{b_{4}}{D_{2}^{2}}\right)=0.085,
$$

with an error $\varepsilon\left(\widetilde{B}_{7}\right)=6.5 \%$.

\subsection{Iterated root approximants}

Employing the iterated root approximants of Sec. 2, we have

$$
\begin{array}{lll}
B_{1}=0.019, & B_{2}=0.039, & B_{3}=0.053, \\
B_{4}=0.061, & B_{5}=0.067, & B_{6}=0.071 .
\end{array}
$$

The error of $B_{6}$ is $\varepsilon\left(B_{6}\right)=-11 \%$.

In this way, for this problem, the corrected root approximants are the most accurate ones.

\section{Strongly interacting fermions}

The ground-state energy of a diluted Fermi gas, using perturbation theory, can be represented $[36,37]$ as an asymptotic expansion

$$
e(g) \simeq a_{0}+a_{1} g+a_{2} g^{2}+a_{3} g^{3}+a_{4} g^{4}
$$

in powers of the dimensionless coupling parameter

$$
g \equiv\left|k_{F} a_{s}\right|
$$

in which $k_{F}$ is a Fermi wave vector and $a_{s}$, scattering length. Here $g \rightarrow 0$ and the coefficients are

$$
\begin{gathered}
a_{0}=\frac{3}{10}, \quad a_{1}=-\frac{1}{3 \pi}, \quad a_{2}=0.055661, \\
a_{3}=-0.00914, \quad a_{4}=-0.018604 .
\end{gathered}
$$

Numerical calculations $[38,39]$ give the strong-coupling limit

$$
\lim _{g \rightarrow \infty} e(g)=0.132
$$

Hence, the strong-coupling exponent is $\beta=0$, while the strong-coupling amplitude is

$$
B=0.132
$$

Padé approximants are not accurate for this problem, the best of them having an error of $30 \%$. 


\subsection{Self-similar factor approximants}

The third-order factor approximant reads as

$$
e_{3}^{*}(g)=\frac{3}{10}\left(1+A_{1} g\right)^{n_{1}}\left(1+A_{2} g\right)^{n_{2}},
$$

under the condition

$$
n_{1}=n_{2}=0 .
$$

This yields the strong-coupling amplitude

$$
B_{3}=\frac{3}{10} A_{1}^{n_{1}} A_{2}^{n_{2}}=0.174,
$$

with an error $\varepsilon\left(B_{3}\right)=32 \%$.

The fourth-order factor approximant can be written as

$$
e_{4}^{*}(g)=\frac{3}{4}+a_{1} g\left(1+A_{3} g\right)^{n_{3}}\left(1+A_{4} g\right)^{n_{4}},
$$

under the condition

$$
1+n_{3}+n_{4}=0 .
$$

Then the strong-coupling amplitude becomes

$$
B_{4}=\frac{3}{10}+a_{1} A_{3}^{n_{3}} A_{4}^{n_{4}}=0.162,
$$

with an error $\varepsilon\left(B_{4}\right)=23 \%$.

\subsection{Corrected factor approximants}

For the correction function

$$
C_{4}(g) \simeq \frac{e_{4}(g)}{e_{3}^{*}(g)} \quad(g \rightarrow 0),
$$

the factor approximant can be represented as

$$
C_{4}^{*}(g)=1+b_{4} g^{4}\left(1+D_{1} g\right)^{-4} .
$$

The corrected factor approximant

$$
\widetilde{e}_{4}(g)=e_{3}^{*}(g) C_{4}^{*}(g)
$$

yields the strong-coupling amplitude

$$
\widetilde{B}_{4}=\frac{3}{10} A_{1}^{n_{1}} A_{2}^{n_{2}}\left(1+\frac{b_{1}}{D_{1}^{4}}\right)=0.1434,
$$

whose error is $\varepsilon\left(\widetilde{B}_{4}\right)=8.6 \%$. 


\subsection{Iterated root approximants}

The iterated root approximants, as defined in Sec. 2, exist for the lowest two approximations, giving

$$
B_{1}=0.098, \quad B_{2}=0.169 .
$$

The higher approximants become complex. The accuracy of $B_{2}$ is characterized by an error $\varepsilon\left(B_{2}\right)=28 \%$.

In this way, the best approximation is provided by the corrected factor approximant (128), with an error of $8.6 \%$.

\section{One-dimensional Bose system}

The one-dimensional Bose gas with local interactions corresponds to the Lieb-Liniger model [40]. The ground-state energy of the gas, in the weak-coupling limit, is described by the expansion

$$
e(g) \simeq g+a_{3} g^{3 / 2}+a_{4} g^{2}+a_{5} g^{5 / 2}
$$

with the coefficients

$$
a_{3}=-\frac{4}{3 \pi}=0.424413, \quad a_{4}=\frac{1.29}{2 \pi^{2}}=0.065352, \quad a_{5}=-0.017201 .
$$

In the strong-coupling limit, one gets the Tonks-Girardeau expression

$$
\lim _{g \rightarrow \infty} e(g)=\frac{\pi^{2}}{3}=B=3.289868 .
$$

More details can be found in the review [41].

\subsection{Simple root approximants}

The simple root approximant

$$
r_{3}^{*}(g)=g\left(\left(1+A_{1} g^{1 / 2}\right)^{n_{1}}+A_{2} g\right)^{-1}
$$

gives the strong-coupling amplitude

$$
B_{3}=\frac{1}{A_{2}}=4.2835
$$

with an error $\varepsilon\left(B_{3}\right)=48 \%$. Certainly, such an accuracy is not sufficient.

\subsection{Iterated root approximants}

Using the iterated root approximants of Sec. 2 , with setting $a_{6}=0$, we get the strongcoupling amplitudes

$$
B_{2}=8.713, \quad B_{3}=4.765, \quad B_{4}=3.2924 \text {. }
$$

The error of the highest-order approximation is $\varepsilon\left(B_{4}\right)=0.08 \%$. This is the best accuracy among the considered approximants. 


\subsection{Inversion of strong-coupling series}

It is worth noting that in those cases when several terms of the strong-coupling expansion are known, it is possible to change the variable and to treat the old strong-coupling expansion as a weak-coupling one in terms of the new variable, and vice versa, the old weak-coupling expansion, as a strong-coupling one. For instance, the strong-coupling expansion for the ground-state energy of the Lieb-Liniger model can be written (see Ref. [41]) in the form

$$
e(g) \simeq \frac{\pi^{2}}{3}\left(1-\frac{4}{g}+\frac{12}{g^{2}}-\frac{32}{g^{3}}+\frac{80}{g^{4}}\right) .
$$

Changing the variable to $x=1 / g$ makes the above series a weak-coupling expansion in terms of $x$. Conversely, Eq. (131) transforms, in terms of $x$, into a strong-coupling expansion [15]. However, possessing quite a number of terms of the strong-coupling limit with respect to the coupling parameter $g$ is a rather rare case. Therefore, in the present paper, we focus on the problem of dealing with the direct coupling parameter, without changing the variable.

\section{Bose-Einstein condensation temperature}

An important problem is the dependence of the Bose-Einstein condensation temperature $T_{c}$ on the gas parameter

$$
\gamma \equiv \rho^{1 / 3} a_{s}
$$

in which $\rho$ is the average atomic density and $a_{s}$, scattering length. For the asymptotically weak interaction, when $\gamma \rightarrow 0$, the relative shift of the critical temperature, with respect to the condensation temperature of the ideal Bose gas,

$$
T_{0}=\frac{2 \pi \hbar^{2}}{m k_{B}}\left[\frac{\rho}{\zeta(3 / 2)}\right]^{2 / 3}
$$

is linear in the gas parameter:

$$
\frac{\Delta T_{c}}{T_{0}} \simeq c_{1} \gamma \quad(\gamma \rightarrow 0)
$$

where

$$
\Delta T_{c} \equiv T_{c}-T_{0}
$$

The coefficient $c_{1}$ has been calculated by a number of different methods (see review articles $[42,43])$. It can be represented as an asymptotic expansion

$$
c_{1}(g) \simeq a_{1} g+a_{2} g^{2}+a_{3} g^{3}+a_{4} g^{4}+a_{5} g^{5}
$$

in powers of an effective coupling parameter [44-46], with the coefficients

$$
\begin{gathered}
a_{1}=0.223286, \quad a_{2}=-0.0661032, \quad a_{3}=0.026446, \\
a_{4}=-0.0129177, \quad a_{5}=0.00729073 .
\end{gathered}
$$


The sought value of $c_{1}$ is the limit

$$
c_{1}=\lim _{g \rightarrow \infty} c_{1}(g)=B
$$

The most accurate calculations of $c_{1}$ have been accomplished by means of the Monte Carlo simulations in Refs. [47-51] and, by using different variants of the optimized perturbation theory [52], in Refs. [44-46,53-55]. Summarizing these results, we have:

$$
\begin{array}{ccc}
c_{1}=1.32 \pm 0.02, & & {[47,48]} \\
c_{2}=1.29 \pm 0.05, & & {[49,50]} \\
c_{1}=1.32 \pm 0.14, & {[51]} \\
c_{1}=1.27 \pm 0.11, & {[45,46]} \\
c_{1}=1.30 \pm 0.03 . & & {[54,55]}
\end{array}
$$

The use of Padé approximants, for summing the asymptotic series (138), does not lead to accurate approximations, the best approximant having an error of $-24 \%$.

\subsection{Self-similar factor approximants}

The direct application of the self-similar factor approximants does not provide high accuracy. Thus, the factor approximant

$$
f_{3}^{*}(g)=a_{1} g\left(1+A_{1} g\right)^{n_{1}}\left(1+A_{2} g\right)^{n_{2}},
$$

under the condition

$$
1+n_{1}+n_{2}=0
$$

gives the amplitude

$$
B_{3}=a_{1} A_{1}^{n_{1}} A_{2}^{n_{2}}=1.025,
$$

which approximates limit (139) with an error $\varepsilon\left(B_{3}\right)=-21 \%$.

\subsection{Simple root approximants}

Simple root approximants also are not accurate enough. For example, the root approximant

$$
r_{2}^{*}(g)=a_{1} g\left(1+A_{1} g\right)^{-1}
$$

yields the amplitude

$$
B_{2}=\frac{a_{1}}{A_{1}}=-\frac{a_{1}}{a_{2}}=0.754
$$

with an error $\varepsilon\left(B_{2}\right)=-42 \%$. And the root approximant

$$
r_{3}^{*}(g)=a_{1} g\left(\left(1+A_{1} g\right)^{n_{1}}+A_{2} g^{2}\right)^{-1 / 2}
$$

gives the amplitude

$$
B_{3}=\frac{a_{1}}{\sqrt{A_{2}}}=0.916
$$

whose accuracy is only sightly better, having an error $\varepsilon\left(B_{3}\right)=-30 \%$. 


\subsection{Iterated root approximants}

The iterated root approximants of Sec. 2 result in the amplitudes

$$
B_{1}=0.754, \quad B_{2}=1.383, \quad B_{3}=0.854 .
$$

The last of these approximations has an error $\varepsilon\left(B_{3}\right)=-34 \%$. The iterated root approximant of the fourth order does not exist, being complex valued.

\subsection{Corrected root approximants}

For the third-order correction function

$$
C_{3}(g) \simeq \frac{a_{1} g+a_{2} g^{2}+a_{3} g^{3}}{r_{2}^{*}(g)} \quad(g \rightarrow 0),
$$

the corresponding root approximant is

$$
C_{3}^{*}(g)=1+b_{2} g^{2}\left(1+D_{1} g\right)^{-2}
$$

The corrected root approximant becomes

$$
\widetilde{r}_{3}(g)=r_{2}^{*}(g) C_{3}^{*}(g)
$$

This gives the amplitude

$$
\widetilde{B}_{3}=-\frac{a_{1}}{a_{2}}\left(1+\frac{b_{1}}{D_{1}^{2}}\right)=0.924
$$

with an error $\varepsilon\left(\widetilde{B}_{3}\right)=-29 \%$.

Increasing the order of the correction function essentially improves the accuracy. Thus, for the fifth-order correction function

$$
C_{5}(g) \simeq \frac{a_{1} g+a_{2} g^{2}+a_{3} g^{3}+a_{4} g^{4}+a_{5} g^{5}}{r_{2}^{*}(g)},
$$

whose root approximant is

$$
C_{5}^{*}(g)=1+b_{2} g^{2}\left(\left(1+D_{1} g\right)^{2}+D_{2} g^{2}\right)^{-1}
$$

we have the corrected root approximant

$$
\widetilde{r}_{5}(g)=r_{2}^{*}(g) C_{5}^{*}(g)
$$

The latter yields the amplitude

$$
\widetilde{B}_{5}=-\frac{a_{1}}{a_{2}}\left(1+\frac{b_{2}}{D_{1}^{2}+D_{2}}\right)=1.29,
$$

which is in the frame of the numerically calculated values of $c_{1}$. 


\subsection{Corrected-iterated root approximants}

The iterated root approximants, considered in subsection 10.3, are not accurate enough. But it is possible to correct them in the spirit of Sec. 2. Let us take, for example, the third-order iterated root approximant

$$
R_{3}(g)=a_{1} g\left(\left(1+A_{1} g\right)^{2}+A_{2} g^{2}\right)^{-1 / 2} .
$$

Introduce the correction function

$$
C_{5}(g) \simeq \frac{a_{1} g+a_{2} g^{2}+a_{3} g^{3}+a_{4} g^{4}+a_{5} g^{5}}{R_{3}(g)},
$$

whose self-similar approximant is

$$
C_{5}^{*}(g)=1+b_{3} g^{3}\left(1+D_{3} g\right)^{-3} .
$$

The corrected-iterated root approximant

$$
R_{5}^{*}(g)=R_{3}(g) C_{5}^{*}(g)
$$

gives the amplitude

$$
B_{5}^{*}=a_{1}\left(A_{1}^{2}+A_{2}\right)^{-1 / 2}\left(1+\frac{b_{3}}{D_{3}^{3}}\right)=1.31,
$$

which is in perfect agreement with numerical calculations for $c_{1}$.

The fifth-order corrected root approximants of subsections 10.4 and 10.5 provide very accurate approximations for the coefficient $c_{1}$.

\section{Conclusion}

In this paper, we have addressed the problem of extrapolating functions, which can be found only in the region of asymptotically weak coupling parameters, to the limit of infinitely strong coupling parameters. The main aim has been, assuming that the strong-coupling exponent is known, to find the strong-coupling amplitude. Several examples are considered, for which Padé approximants do not provide good accuracy or are not applicable at all. For these problems, we employ the self-similar approximation theory, comparing different variants of self-similar approximants. Two new classes of self-similar approximants are suggested, the corrected approximants and iterated approximants.

Comparing the accuracy of different self-similar approximants for different problems, we find the following. For the Debye-Hückel function (Sec. 3), the highest accuracy is provided by the iterated root approximants. For the quartic anharmonic oscillator (Sec. 4), the best are the self-similar factor approximants. For the polymer chain (Sec. 5), all considered variants of the self-similar approximants provide almost the same accuracy. For the oscillating fluid string (Sec. 6), the best are the corrected root approximants. For the fluctuating fluid membrane (Sec. 7), the corrected root approximants are also the most accurate. For strongly interacting fermions (Sec. 8), the best are the corrected factor approximants. For the one-dimensional Bose system (Sec. 9), the iterated root approximants can give the best accuracy, though with a large dispersion between the different-order approximants. For the 
Bose-Einstein condensation temperature (Sec. 10), the corrected root approximants give the best accuracy, yielding the results that are in very good agreement with the known numerical calculations.

The considered examples illustrate that the self-similar approximants provide a versatile tool for extrapolation problems.

\section{Acknowledgments}

The authors are grateful to E.P. Yukalova for useful discussions. One of the authors (V.I.Y.) appreciates financial support from the Russian Foundation for Basic Research. 


\section{References}

[1] H. Kleinert, Path Integrals (World Scientific, Singapore, 2006).

[2] V.I. Yukalov, Phys. Rev. A 42, 3324 (1990).

[3] V.I. Yukalov, Physica A 167, 833 (1990).

[4] V.I. Yukalov, J. Math. Phys. 32, 1235 (1991).

[5] V.I. Yukalov, J. Math. Phys. 33, 3994 (1992).

[6] V.I. Yukalov, E.P. Yukalova, Physica A 198, 573 (1993).

[7] V.I. Yukalov, E.P. Yukalova, Physica A 206, 553 (1994).

[8] V.I. Yukalov, E.P. Yukalova, Physica A 225, 336 (1996).

[9] V.I. Yukalov, S. Gluzman, Phys. Rev. Lett. 79, 333 (1997).

[10] S. Gluzman, V.I. Yukalov, Phys. Rev. E 58, 4197 (1998).

[11] V.I. Yukalov, E.P. Yukalova, S. Gluzman, Phys. Rev. A 58, 96 (1998).

[12] V.I. Yukalov, S. Gluzman, Physica A 273, 401 (1999).

[13] G.A. Baker, P. Graves-Moris, Padé Approximants (Cambridge University, Cambridge, 1996).

[14] S. Gluzman, V.I. Yukalov, J. Math. Chem. 39, 47 (2006).

[15] V.I. Yukalov, E.P. Yukalova, S. Gluzman, J. Math. Chem. in press.

[16] V.I. Yukalov, S. Gluzman, Phys. Rev. E 55, 6552 (1997).

[17] V.I.Yukalov, S. Gluzman, Phys. Rev. E 58, 1359 (1998).

[18] S. Gluzman, V.I. Yukalov, D. Sornette, Phys. Rev. E 67, 026109 (2003)

[19] V.I. Yukalov, S. Gluzman, D. Sornette, Physica A 328, 409 (2003).

[20] V.I. Yukalov, E.P. Yukalova, Phys. Lett. A 368, 341 (2007).

[21] E.P. Yukalova, V.I. Yukalov, S. Gluzman, Ann. Phys. (NY) 323, 3074 (2008).

[22] V.I. Yukalov, E.P. Yukalova, Chaos Solit. Fract. 14, 839 (2002).

[23] E.A. Guggenheim, Thermodynamics (North-Holland, Amsterdam, 1957).

[24] L.D. Landau, E.M. Lifshitz Statistical Physics (Butterworth-Heinemann, Oxford, 2000).

[25] F.T. Hioe, D. McMillen, E.W. Montroll, Phys. Rep. 43, 307 (1978).

[26] V.I. Yukalov, E.P. Yukalova, Laser Phys. 5, 154 (1995). 
[27] M. Muthukumar, B.G. Nickel, J. Chem. Phys. 80, 5839 (1984).

[28] M. Muthukumar, B.G. Nickel, J. Chem. Phys. 86, 460 (1987).

[29] B. Li, N. Madras, A.D. Sokal, J. Stat. Phys. 80, 661 (1995).

[30] U. Seifert, Adv. Phys. 46, 13 (1997).

[31] S.F. Edwards, Proc. Roy. Soc. London 85, 613 (1965).

[32] H. Kleinert, Phys. Lett. A 257, 269 (1999).

[33] B. Kastening, Phys. Rev. E 66, 061102 (2002).

[34] B. Kastening, Phys. Rev. E 73, 011101 (2006).

[35] G. Gompper, D.M. Kroll, Eur. Phys. Lett. 9, 59 (1989).

[36] G.A. Baker, Phys. Rev. C 60, 054311 (1999).

[37] W. Ketterle, M.W. Zwierlein, Riv. Nuovo Cimento 31, 247 (2008).

[38] J. Carlson, S.Y. Chang, V.R. Pandharipande, K.E. Schmidt, Phys. Rev. Lett. 91, 050401 (2003).

[39] G.E. Astrakharchik, J. Boronat, J. Casulleras, S. Giorgini, Phys. Rev. Lett. 93, 200404 (2004).

[40] E.H. Lieb, W. Liniger, Phys. Rev. 130, 1605 (1963).

[41] V.I. Yukalov, M.D. Girardeau, Laser Phys. Lett. 2, 375 (2005).

[42] J.O. Andersen, Rev. Mod. Phys. 76, 599 (2004)

[43] V.I. Yukalov, Laser Phys. Lett. 1, 435 (2004).

[44] B. Kastening, Laser Phys. 14, 586 (2004).

[45] B. Kastening, Phys. Rev. A 69, 043613 (2004).

[46] B. Kastening, Phys. Rev. A 70, 043621 (2004).

[47] P. Arnold, G. Moore, Phys. Rev. Lett. 87, 120401 (2001).

[48] P. Arnold, G. Moore, Phys. Rev. E 64, 066113 (2001).

[49] V.A. Kashurnikov, N. Prokofiev, B. Svistunov, Phys. Rev. Lett. 87, 120402 (2001).

[50] N. Prokofiev, B. Svistunov, Phys. Rev. Lett. 87, 160601 (2001).

[51] K. Nho, D.P. Landau, Phys. Rev. A 70, 053614 (2004).

[52] V.I. Yukalov, Moscow Univ. Phys. Bull. 31, 10 (1976). 
[53] J.L. Kneur, M.B. Pinto, R.O. Ramos, Phys. Rev. Lett. 89, 210403 (2002).

[54] J.L. Kneur, A. Neveu, M.B. Pinto, Phys. Rev. A 69, 053624 (2004).

[55] J.L. Kneur, M.B. Pinto, Phys. Rev. A 71, 033613 (2005). 